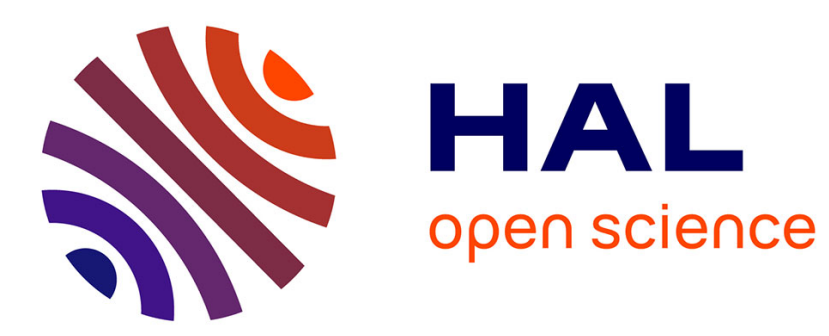

\title{
The speed of a branching system of random walks in random environment
}

Alexis Devulder

\section{To cite this version:}

Alexis Devulder. The speed of a branching system of random walks in random environment. Statistics and Probability Letters, 2009, 77 (18), pp.1712. 10.1016/j.spl.2007.04.010 . hal-00594446

\section{HAL Id: hal-00594446 \\ https://hal.science/hal-00594446}

Submitted on 20 May 2011

HAL is a multi-disciplinary open access archive for the deposit and dissemination of scientific research documents, whether they are published or not. The documents may come from teaching and research institutions in France or abroad, or from public or private research centers.
L'archive ouverte pluridisciplinaire HAL, est destinée au dépôt et à la diffusion de documents scientifiques de niveau recherche, publiés ou non, émanant des établissements d'enseignement et de recherche français ou étrangers, des laboratoires publics ou privés. 


\section{Author's Accepted Manuscript}

The speed of a branching system of random walks in random environment

Alexis Devulder

PII:

S0167-7152(07)00156-3

DOI: doi:10.1016/j.spl.2007.04.010

Reference: STAPRO 4659

To appear in: $\quad$ Statistics \& Probability Letters

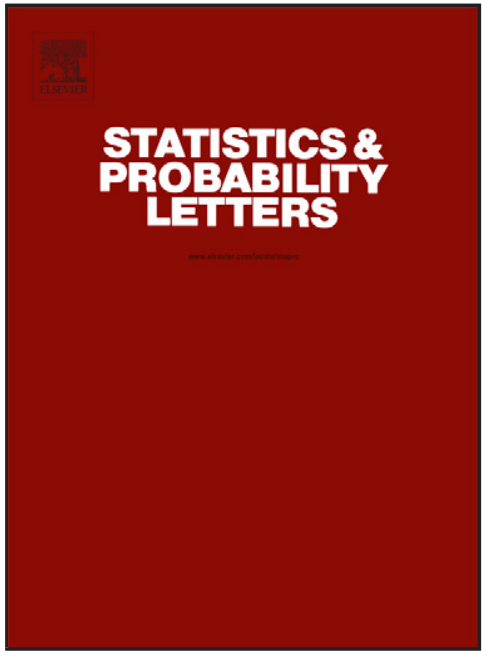

www.elsevier.com/locate/stapro

Received date: $\quad 20$ April 2006

Revised date: 5 February 2007

Accepted date: $\quad 10$ April 2007

Cite this article as: Alexis Devulder, The speed of a branching system of random walks in random environment, Statistics \& Probability Letters (2007), doi:10.1016/j.spl.2007.04.010

This is a PDF file of an unedited manuscript that has been accepted for publication. As a service to our customers we are providing this early version of the manuscript. The manuscript will undergo copyediting, typesetting, and review of the resulting galley proof before it is published in its final citable form. Please note that during the production process errors may be discovered which could affect the content, and all legal disclaimers that apply to the journal pertain. 


\title{
The speed of a branching system of random walks in random environment
}

\author{
Alexis Devulder*
}

February 5, 2007

\begin{abstract}
We consider a branching system of random walks in random environment in $\mathbb{Z}$, for which extinction is possible. We study the speed of the rightmost particle, conditionally on the survival of the branching process.
\end{abstract}

KEY WoRDS: Random walk in random environment, branching random walk, Galton-Watson tree.

AMS (2000) Classification: 60J80, 60K37.

\section{Introduction}

Let $\left(\omega_{i}\right)_{i \in \mathbb{Z}}$ be a collection of independent and identically distributed random variables, taking values in $(0,1)$. For any realization of the environment $\omega:=\left(\omega_{i}\right)_{i \in \mathbb{Z}}$, we define a random walk $\left(X_{n}\right)_{n \in \mathbb{N}:=\{0,1,2, \ldots\}}$ which is a Markov chain with $X_{0}=0$ such that for $n \geq 0$ and $i \in \mathbb{Z}$,

$$
P_{\omega}\left(X_{n+1}=i+1 \mid X_{n}=i\right)=\omega_{i}, \quad P_{\omega}\left(X_{n+1}=i-1 \mid X_{n}=i\right)=1-\omega_{i} .
$$

We call $P_{\omega}$ the quenched law. If $\eta$ denotes the law of the environment $\left(\omega_{i}\right)_{i \in \mathbb{Z}}$, we also let

$$
\mathbb{P}(.):=\int P_{\omega}(.) \eta(\mathrm{d} \omega)
$$

and call the resulting law the annealed law. This model is known as "random walk in random environment", abbreviated as RWRE. It exhibits some unusual properties, in both annealed and quenched cases (see for example Zeitouni, 2004).

For technical reasons, we furthermore assume that there exists $\delta>0$ such that $\omega_{0} \in(\delta, 1-\delta) \eta-$ a.s. This is needed to apply the results on large deviations for RWRE, see Section 2.2 .

Let $\rho_{i}:=\frac{1-\omega_{i}}{\omega_{i}}, i \in \mathbb{Z}$. Solomon (1975) proved that the RWRE is $\eta$-a.s. recurrent if and only if $\int \log \rho_{0}(\omega) \eta(\mathrm{d} \omega)=0$. In the transient case, $\lim _{n \rightarrow \infty} X_{n}=+\infty \mathbb{P}-$ a.s. if $\int \log \rho_{0}(\omega) \eta(\mathrm{d} \omega)<0$, and $\lim _{n \rightarrow \infty} X_{n}=-\infty \mathbb{P}$-a.s. if $\int \log \rho_{0}(\omega) \eta(\mathrm{d} \omega)>0$.

* Laboratoire de Mathématiques de Versailles, Université de Versailles-Saint Quentin en Yvelines, 45 avenue des Etats-Unis, 78035 Versailles cedex, France. E-mail: devulder@math.uvsq.fr. 
Without loss of generality, we assume that $\int \log \rho_{0}(\omega) \eta(\mathrm{d} \omega) \geq 0$ (otherwise, we only have to replace $\left(\omega_{i}\right)_{i \in \mathbb{Z}}$ by $\left.\left(1-\omega_{i}\right)_{i \in \mathbb{Z}}\right)$.

We are interested in a model of branching system of random walks in random environment, where the particles reproduce with a fixed reproduction law, but move as random walks in random environment. This is the analogue in random environment of the model of branching random walks; the latter being well studied in the literature, see for example Révész (1994). We mention that our model is very much different from the socalled "branching random walk in random environment" (see for example Greven and den Hollander (1992), where the reproduction law of the particles depends on their locations while the transition probabilities are the same everywhere.

Here is a description of our model. Let $p_{k} \geq 0$ for all $k \in \mathbb{N}$, such that $\sum_{k \in \mathbb{N}} p_{k}=1$. For each given environment, the particle system behaves like this:

- At time $n=0$ there is only one particle, located at 0 .

- At time $n=1$ the particle moves to 1 with probability $\omega_{0}$, or to -1 with probability $1-\omega_{0}$. Arriving at the new location, it gives birth to $k$ offspring with probability $p_{k}$, and dies.

- At time $n=2$ each particle moves independently (to a neighbouring site), according to the probabilities in (1). Then it produces new offspring independently, with the same reproduction law as before, and dies.

- Iterating this procedure we obtain a branching system of random walks in random environment.

We notice that the branching process, denoted by $\Gamma$, is a Galton-Watson process. We assume that it is supercritical, i.e., the expected amount of offspring of each particle, denoted by $m$, lies in $(1,+\infty)$. We also assume that the amount of offspring has a finite variance $\sigma^{2}$. We focus on this model throughout the paper. Notice that we allow the particles to have no offspring, hence the extinction of the branching process is possible. This considerably complicates the situation. A similar model has already been studied by Comets et al. (1998) on the half line $\mathbb{N}$. See also Comets et al. (2005) for multidimensional random walks in random environment. However, for these models, the size of the population cannot decrease.

In the case $\int \log \rho_{0}(\omega) \eta(\mathrm{d} \omega)>0$, there is for our model a competition between the environment, pushing the particles to $-\infty$, and the branching process, which creates new particles and then increases the possibility that some particles go very far on the right. It is therefore natural to study the asymptotic behaviour of the rightmost particle.

Let $\omega_{\max }:=\sup \left\{x, x \in \operatorname{Supp} \omega_{0}\right\}$ and

$$
m_{c}:= \begin{cases}1 & \text { if } \frac{1}{2} \in \operatorname{Supp} \omega_{0}, \\ \frac{1}{2}\left[\omega_{\max }\left(1-\omega_{\max }\right)\right]^{-1 / 2} & \text { if Supp } \omega_{0} \subset\left[\delta, \frac{1}{2}\right) .\end{cases}
$$

Moreover, notice that $m_{c}=\exp \left(I_{\eta}^{q}(0)\right)$, and that $m_{c}$ depends only on the law $\eta$ of the environment.

The main result of this paper is the existence of a non-zero speed for the rightmost particle if $m \neq m_{c}$. More precisely, $\mathbb{P}$-almost surely when the system of particles survives,

(i) if $m<m_{c}$, then the rightmost particle goes to $-\infty$ with a negative speed;

(ii) if $m>m_{c}$, then the rightmost particle goes to $+\infty$ with a positive speed.

In the model of Comets et al. (1998), the reproduction law also depends on the location, and each particle has almost surely at least one offspring. Hence the branching system always survives. Their result shows that $m_{c}$ is a critical value for our model in this case. If $m>m_{c}$, there is infinitely often at least one particle with positive location, and if $m \leq m_{c}$, there is no particle in $\mathbb{N}$ at time $n$ for $n$ large enough.

By proving Theorem 1.1 below, we show again as a by-product that $m_{c}$ is a critical value in our setting. Notice that our proof hinges upon a different method, using in particular large deviations for random walks in random environment. In our model, the reproduction law is fixed and does not depend on the location. However, we allow the particles to have no offspring.

Let $m_{n}^{*}$ denote the location of the rightmost particle at time $n$.

Theorem 1.1 Assume $\int \log \rho_{0}(\omega) \eta(\mathrm{d} \omega) \geq 0$, and let $\Gamma$ be the Galton-Watson process governing the branching system. 
(i) If $1<m<m_{c}$, then

$$
\mathbb{P}\left(\limsup _{n \rightarrow \infty} \frac{m_{n}^{*}}{n}<0 \mid \Gamma \text { survives }\right)=1 .
$$

(ii) If $m>m_{c}$, then

$$
\mathbb{P}\left(\liminf _{n \rightarrow \infty} \frac{m_{n}^{*}}{n}>0 \mid \Gamma \text { survives }\right)=1 .
$$

In some cases, we have $m_{c}=1$, which means we are always in situation (ii). It is for example the case when the RWRE is recurrent (i.e., if $\int \log \rho_{0}(\omega) \eta(\mathrm{d} \omega)=0$ ). Moreover, for $\Delta \subset \mathbb{Z}$, we denote by $\lambda(\Delta, k)$ the number of particles located in $\Delta$ at time $k$. We prove,

Proposition 1.2 If $m=m_{c}$, then $E_{\omega}(\lambda(\mathbb{N}, 2 k)) \leq 1 \quad \forall k \in \mathbb{N}, \quad \mathbb{P}$-a.s. and

$$
\mathbb{P}\left(\limsup _{n \rightarrow \infty} \frac{m_{n}^{*}}{n} \leq 0 \mid \Gamma \text { survives }\right)=1 .
$$

Thus there are very few particles (if any) in $\mathbb{N}$ in the critical case $m=m_{c}$. Moreover, Gantert et al. (2004) prove (in a more general setting) that in this critical case, when there is always at least one offspring particle, the system is "transient", that is, $m_{n}^{*} \rightarrow-\infty$.

The article is organized as follows. In Section 2, we give the precise mathematical formulation of the model and present some preliminary results. The proof for the case $m<m_{c}$ is provided in Section 3 . Section 4, which is the heart of this paper, consists of the proof for the case $m>m_{c}$. We prove Proposition 1.2 in Section 5. Finally, we give some additional comments in Section 6.

\section{Preliminaries}

This section is divided into two parts. The first one consists of a formal definition of the model, whereas the second one deals with large deviations for the RWRE.

\subsection{Precise formulation of the model}

We now give a more formal definition of the model and precise some notation.

Recall that the random variables $\omega_{i}$ are assumed to be independent and identically distributed, with joint distribution $\eta$.

Following Révész (1994) (who studied the case of usual branching random walks), we introduce the

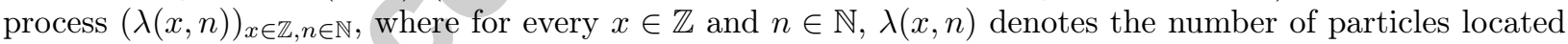
at $x$ at time $n$. With this notation, $m_{n}^{*}=\max \{x \in \mathbb{Z}, \lambda(x, n)>0\}$. Let $(Z(x, n, \mu), x \in \mathbb{Z}, n \in \mathbb{N}, \mu \in \mathbb{N})$ be independent random variables, independent of $\omega$, such that $\mathbb{P}(Z(x, n, \mu)=k)=p_{k}$ for all $(x, n, \mu, k) \in \mathbb{Z} \times \mathbb{N}^{3}$. It helps to bear in mind that $Z(x, n, \mu)$ stands for the number of children of the $\mu$-th particle born at location $x$ at time $n$.

Let $(X(x, n, \mu), x \in \mathbb{Z}, n \in \mathbb{N}, \mu \in \mathbb{N})$ be independent random variables having the common uniform law on $[0,1]$, which are independent of $\left(\omega_{i}\right)_{i \in \mathbb{Z}}$ and $(Z(x, n, \mu), x \in \mathbb{Z}, n \in \mathbb{N}, \mu \in \mathbb{N})$. Conditionally on the environment, the movement of the $\mu$-th particle born at location $x$ at time $n$ depends only on $X(x, n, \mu)$.

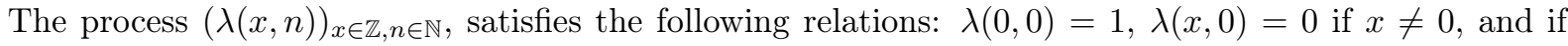
$n \geq 0$ and $x \in \mathbb{Z}$,

$$
\lambda(x, n+1)=\sum_{\mu=1}^{\lambda(x-1, n)} \mathbf{1}_{\left\{X(x-1, n, \mu) \leq \omega_{x-1}\right\}} Z(x-1, n, \mu)+\sum_{\mu=1}^{\lambda(x+1, n)} \mathbf{1}_{\left\{X(x+1, n, \mu)>\omega_{x+1}\right\}} Z(x+1, n, \mu) .
$$


The branching process consituted of the particles in the system is denoted by $\Gamma$, as in the Introduction. We still denote by $P_{\omega}$ the law of the particle system conditionally on the environment $\omega$. As for RWRE we define $\mathbb{P}():.=\int P_{\omega}(.) \eta(\mathrm{d} \omega)$. Moreover, $E_{\omega}$ and $\mathbb{E}$ stand for the expectations with respect to $P_{\omega}$ and $\mathbb{P}$ respectively. We also let $\mathbb{P}^{x}$ and $\mathbb{E}^{x}$ (respectively $P_{\omega}^{x}$ and $E_{\omega}^{x}$ ) be the probability and expectation of the system (respectively conditionally on $\omega$ ) when the root is located at $x$ instead of 0 .

We need some more notation. For every $(x, y) \in \mathbb{Z}^{2}, \Delta \subset \mathbb{Z}$ and $(n, p) \in \mathbb{N}^{2}$, let

$$
p_{\omega}(x \rightsquigarrow y, n):=P_{\omega}\left(X_{p+n}=y \mid X_{p}=x\right), \quad p_{\omega}(x \rightsquigarrow \Delta, n):=P_{\omega}\left(X_{p+n} \in \Delta \mid X_{p}=x\right) .
$$

Moreover, for any environment $\omega$ and any branching system of RWRE, we write

$$
\begin{aligned}
\mathcal{F}_{\omega}(N) & :=\sigma(\lambda(x, n), x \in \mathbb{Z}, 0 \leq n \leq N), & & N \in \mathbb{N}, \\
f_{\omega}(x, N, n) & :=m^{N-n} \sum_{y \in \mathbb{Z}} \lambda(y, n) p_{\omega}(y \rightsquigarrow x, N-n), & & N \in \mathbb{N}, x \in \mathbb{Z}, n \in\{0,1, \ldots N\} .
\end{aligned}
$$

We can now prove a couple of lemmas, which are simple adaptations from results of Révész (1994):

Lemma 2.1 We have, for $N \in \mathbb{N}$,

$$
\forall 0 \leq n \leq N, \forall x \in \mathbb{Z}, \quad E_{\omega}\left(\lambda(x, N) \mid \mathcal{F}_{\omega}(n)\right)=f_{\omega}(x, N, n) \quad \mathbb{P} \text {-a.s. }
$$

Proof: In the case $N=n$, for each $x \in \mathbb{Z}$,

$$
E_{\omega}\left(\lambda(x, N) \mid \mathcal{F}_{\omega}(n)\right)=\lambda(x, N)=\sum_{y \in \mathbb{Z}} \lambda(y, N) p_{\omega}(y \rightsquigarrow x, 0)=f_{\omega}(x, N, N) .
$$

Thus (4) is true when $N=n$ and in particular when $N=n=0$.

We deal with the general case by induction. Suppose that for some $N \in \mathbb{N}$, formula (4) is true for all $0 \leq n \leq N$ and $x \in \mathbb{Z}$. Therefore, if $n<N+1$ (the case $n=N+1$ has already been treated),

$$
\begin{aligned}
& E_{\omega}\left(\lambda(x, N+1) \mid \mathcal{F}_{\omega}(n)\right) \\
= & E_{\omega}\left(\sum_{\mu=1}^{\lambda(x-1, N)} \mathbf{1}_{\left\{X(x-1, N, \mu) \leq \omega_{x-1}\right\}} Z(x-1, N, \mu)+\sum_{\mu=1}^{\lambda(x+1, N)} \mathbf{1}_{\left\{X(x+1, N, \mu)>\omega_{x+1}\right\}} Z(x+1, N, \mu) \mid \mathcal{F}_{\omega}(n)\right) \\
= & m \omega_{x-1} E_{\omega}\left(\lambda(x-1, N) \mid \mathcal{F}_{\omega}(n)\right)+m\left(1-\omega_{x+1}\right) E_{\omega}\left(\lambda(x+1, N) \mid \mathcal{F}_{\omega}(n)\right),
\end{aligned}
$$

which is by induction $\mathbb{P}$-a.s. equal to

$$
\begin{aligned}
& m \omega_{x-1} f_{\omega}(x-1, N, n)+m\left(1-\omega_{x+1}\right) f_{\omega}(x+1, N, n) \\
= & m m^{N-n} \sum_{y \in \mathbb{Z}}\left[\omega_{x-1} p_{\omega}(y \rightsquigarrow x-1, N-n)+\left(1-\omega_{x+1}\right) p_{\omega}(y \rightsquigarrow x+1, N-n)\right] \lambda(y, n) .
\end{aligned}
$$

Hence,

$$
E_{\omega}\left(\lambda(x, N+1) \mid \mathcal{F}_{\omega}(n)\right)=m^{N+1-n} \sum_{y \in \mathbb{Z}} \lambda(y, n) p_{\omega}(y \rightsquigarrow x, N+1-n)=f_{\omega}(x, N+1, n) .
$$

This yields the desired conclusion. 
As a consequence, we obtain

Lemma 2.2 We have, for $N \in \mathbb{N}$ and $x \in \mathbb{Z}$,

$$
E_{\omega}(\lambda(x, N))=m^{N} p_{\omega}(0 \rightsquigarrow x, N) \quad \mathbb{P} \text {-a.s. }
$$

Proof: For $N \in \mathbb{N}$ and $x \in \mathbb{Z}$, Lemma 2.1 gives $\mathbb{P}$-a.s.,

$$
E_{\omega}[\lambda(x, N)]=E_{\omega}\left[\lambda(x, N) \mid \mathcal{F}_{\omega}(0)\right]=f_{\omega}(x, N, 0)=m^{N} \sum_{y \in Z} \lambda(y, 0) p_{\omega}(y \rightsquigarrow x, N)=m^{N} p_{\omega}(0 \rightsquigarrow x, N)
$$

since at time 0 there is only one particle, which is at location 0 .

\section{$2.2 \quad$ Large deviations}

It is known (Solomon, 1975) that the $\operatorname{RWRE}\left(X_{n}\right)_{n \in \mathbb{N}}$ satisfies a law of large numbers: there exists $v \in[-1,1]$ such that $\lim _{n \rightarrow \infty} X_{n} / n=v \mathbb{P}$-a.s. Under our assumption $\int \log \rho_{0}(\omega) \eta(\mathrm{d} \omega) \geq 0$, we have $v \in[-1,0]$.

The RWRE $\left(X_{n}\right)_{n \in \mathbb{N}}$ moreover satisfies a quenched large deviation principle with deterministic, convex and continuous rate function $I_{\eta}^{q}$ (see Greven and den Hollander, 1994). This means there exists a nonnegative convex function $I_{\eta}^{q}$ such that $\eta$-a.s. for any measurable set $A$,

$$
-\inf _{x \in A^{\circ}} I_{\eta}^{q}(x) \leq \liminf _{n \rightarrow \infty} \frac{1}{n} \log P_{\omega}\left(X_{n} / n \in A\right) \leq \limsup _{n \rightarrow \infty} \frac{1}{n} \log P_{\omega}\left(X_{n} / n \in A\right) \leq-\inf _{x \in \bar{A}} I_{\eta}^{q}(x),
$$

where $A^{\circ}$ denotes the interior of $A$ and $\bar{A}$ is the closure of $A$. In case $\int \log \rho_{0}(\omega) \eta(\mathrm{d} \omega)>0$, Comets et al. (2000, Cases $E$ and $F$ in their Proposition 2) gave the shape of the rate function $I_{\eta}^{q}$. Let $\omega_{\min }:=\inf \{x, x \in$ Supp $\left.\omega_{0}\right\}$. If $\omega_{\min } \leq \frac{1}{2} \leq \omega_{\max }$, then $I_{\eta}^{q}(0)=0$. If $\omega_{\max }<\frac{1}{2}$, then $v<0$ and $I_{\eta}^{q}(v)=0$, and $I_{\eta}^{q}$ is strictly increasing on $[v, 1]$.

In the recurrent case $\int \log \rho_{0}(\omega) \eta(\mathrm{d} \omega)=0$, we simply have $v=0$ and $I_{\eta}^{q}(0)=0$ (Comets et al. 2000, Case A of Proposition 2).

The above was proved in Comets et al. (2000), under the additional assumption that $\eta$ is non degenerate (i.e., it is not concentrated on a single point). However, it holds trivially if $\eta$ is degenerate, which leads to the case of usual random walk. Notice that Comets et al. (2000) ask $\eta$ to be a product measure such that there exists $\delta>0, \omega_{0} \in(\delta, 1-\delta) \eta$-a.s.

\section{Proof of Theorem 1.1; case $m<m_{c}$}

In this section, we study the case $1<m<m_{c}=\exp \left(I_{\eta}^{q}(0)\right)$. This implies that $I_{\eta}^{q}(0)>0$, thus $I_{\eta}^{q}$ is strictly increasing on $(v, 1]$ with $v<0$. As a consequence, since $I_{\eta}^{q}$ is a continuous function, there exist $\alpha>0$ and $\varepsilon>0$ such that $\log m<I_{\eta}^{q}(-\alpha)-\varepsilon$. According to $(5)$, we obtain for the RWRE $\left(X_{n}\right)_{n \in \mathbb{N}}, \mathbb{P}-$ a.s. for $n$ large enough,

$$
P_{\omega}\left(X_{n} \geq-\alpha n\right) \leq \exp \left[-\left(I_{\eta}^{q}(-\alpha)-\varepsilon\right) n\right] .
$$

As a consequence, recalling Lemma 2.2, we have $\mathbb{P}$-a.s. for all large $n$,

$$
P_{\omega}\{\lambda[(-\alpha n,+\infty), n] \geq 1\} \leq E_{\omega}\left[\sum_{x=-\alpha n}^{\infty} \lambda(x, n)\right]=m^{n} P_{\omega}\left[X_{n} \geq-\alpha n\right] \leq \exp \left\{\left[\log m-I_{\eta}^{q}(-\alpha)+\varepsilon\right] n\right\}
$$


Since $\log m-I_{\eta}^{q}(-a)+\varepsilon<0$, we obtain

$$
\sum_{n \geq 0} P_{\omega}\{\lambda((-\alpha n,+\infty), n) \geq 1\}<\infty \quad \mathbb{P}-\text { a.s. }
$$

By the Borel-Cantelli lemma, $P_{\omega}$-a.s. for $n$ large enough, there is no particle in $(-\alpha n,+\infty)$. That is, $\mathbb{P}-$ a.s., $m_{n}^{*}<-\alpha n$ for $n$ large enough (with $\sup \emptyset=-\infty$ by convention), which gives the first part of Theorem 1.1. $\square$

\section{Proof of Theorem 1.1; case $m>m_{c}$}

We now consider the case $m>m_{c}:=\exp \left(I_{\eta}^{q}(0)\right)$, and we prove that if $\Gamma$ survives, the rightmost particle in the system goes to $+\infty$ with a positive speed.

The proof is divided into three steps. (a) We first construct a supercritical Galton-Watson tree $T$ whose vertices of the $n$-th generation are particles which are at a positive location at time $n k_{\omega}\left(k_{\omega}\right.$ is an integer defined below). When $T$ survives, which occurs with a positive probability, there is an exponential number of particles in $\mathbb{N}$ at times $n k_{\omega}, n \in \mathbb{N}$. (b) Some of the particles originated from $T$ will go far enough. (c) Although $T$ only has a positive survival probability, there are always particles going very far, as long as the branching process $\Gamma$ survives.

For the sake of clarity, the three parts are presented in distinct subsections.

\subsection{Construction of $T$}

The basic idea in the construction goes back to Hammersley (1974), Kingman (1975), and Biggins (1977). See also Peres (1999, Theorem 18.3) for example.

Recall that $\log m>\log m_{c}=I_{\eta}^{q}(0)$, and fix $\varepsilon>0$ such that $\log m>I_{\eta}^{q}(0)+\varepsilon$. According to (5), we have $\mathbb{P}$-a.s.,

$$
\exists n_{\omega} \in \mathbb{N}^{*}, \forall n \geq n_{\omega}, \quad P_{\omega}\left(X_{n} \geq 0\right) \geq \exp \left[-\left(I_{\eta}^{q}(0)+\varepsilon\right) n\right] .
$$

Fix such an environment $\omega$, and consider our branching system of particles. By Lemma 2.2 , for $k \geq n_{\omega}$,

$$
E_{\omega}\left(\sum_{x \in \mathbb{N}} \lambda(x, k)\right)=m^{k} P_{\omega}\left(X_{k} \geq 0\right) \geq \exp \left[\left(\log m-I_{\eta}^{q}(0)-\varepsilon\right) k\right] .
$$

Since $\log m>I_{\eta}^{q}(0)+\varepsilon$, we can fix an even integer $k_{\omega}>0$ such that

$$
E_{\omega}\left(\sum_{x \in \mathbb{N}} \lambda\left(x, k_{\omega}\right)\right):=\Lambda_{\omega}>2 .
$$

We will be working from now on with these fixed constants $k_{\omega}$ and $\Lambda_{\omega}$.

We now build recursively a sequence of random variables $\left(Y_{n}\right)_{n \in \mathbb{N}}$ such that at each time $n k_{\omega}, n \in \mathbb{N}$, there are at least $Y_{n}$ particles located in $\mathbb{N}$ :

- At time 0 , there is only one particle, which is located at 0 . We set $Y_{0}=1$.

- Let $Y_{1}$ be the number of particles located in $\mathbb{N}$ at time $k_{\omega}$, that is, $Y_{1}=\lambda\left(\mathbb{N}, k_{\omega}\right)$.

- Suppose that at time $n k_{\omega}$ there are at least $Y_{n}$ particles in $\mathbb{N}$. Let $x_{1}, x_{2}, \ldots, x_{Y_{n}}$ be the locations of $Y_{n}$ such particles. We only consider these $Y_{n}$ particles and ignore all the other particles which are possibly surviving at time $n k_{\omega}$. By a natural coupling argument, it is easily seen that the number of 
particles located in $\mathbb{N}$ at time $(n+1) k_{\omega}$ and generated by these $Y_{n}$ particles, is greater than or equal to the number of particles located in $\mathbb{N}$ at time $(n+1) k_{\omega}$ and generated by $Y_{n}$ particles all of which are located at 0 (instead of $\left.\left(x_{i}\right)\right)$ at time $n k_{\omega}$. Thus, at time $(n+1) k_{\omega}$, there are at least $Y_{n+1}$ particles in $\mathbb{N}$, where

$$
Y_{n+1}:=\sum_{i=1}^{Y_{n}} X_{n, i}
$$

and the variables $\left(X_{n, i}\right)$ have the same law as $Y_{1}$, and are independent (given $\omega$ ). Moreover, the variables $\left(X_{n, i}\right)$ are independent of $\mathcal{F}_{\omega}\left(n k_{\omega}\right)$.

We denote by $T$ the resulting Galton-Watson tree, and by $Y_{n}$ the cardinality of its $n$-th generation. According to (7), $E_{\omega}\left(Y_{1}\right)=\Lambda_{\omega}>2$. Thus $T$ is supercritical; its extinction probability is less than 1 .

We are now ready to prove the main technical estimate in this subsection.

Lemma 4.1 We have, $\mathbb{P}$-almost surely,

$$
\lim _{n \rightarrow \infty} P_{\omega}\left(\bigcap_{\ell \geq n}\left\{Y_{\ell} \geq 2^{\ell}\right\}\right)>0
$$

Proof: Consider the original particle system. The amount of offspring of each particle has a finite variance. Therefore the total number of particles at time $k_{\omega}$, denoted by $B\left(k_{\omega}\right)$, satisfies $E_{\omega}\left(B\left(k_{\omega}\right)^{2}\right)<\infty$. As a consequence, we have $E_{\omega}\left(\left(Y_{1}\right)^{2}\right)<\infty$. Since $E_{\omega}\left(Y_{1}\right)=\Lambda_{\omega}>2$ (see (7)), which is greater than 1 , there exists a random variable $W_{\omega}$, satisfying $P_{\omega}\left(W_{\omega}>0\right)>0 \mathbb{P}$-a.s., such that $Y_{n} \sim_{n \rightarrow \infty}\left(\Lambda_{\omega}\right)^{n} W_{\omega} \mathbb{P}-$ a.s. (see Athreya and Ney, 1972, p. 9). Since $\Lambda_{\omega}>2$, we have $\left(\Lambda_{\omega}\right)^{n} W_{\omega} \geq 2^{n}$ for $n$ large enough if $W_{\omega}>0$. Accordingly,

$$
\lim _{n \rightarrow \infty} P_{\omega}\left(\bigcap_{\ell \geq n}\left\{Y_{\ell} \geq 2^{\ell}\right\}\right)=P_{\omega}\left(W_{\omega}>0\right)>0 \quad \mathbb{P}-\text { a.s. }
$$

as desired.

\subsection{Particles going to infinity}

This subsection is devoted to prove the following lemma.

Lemma 4.2 Let $m_{n}^{*}$ denote as before the location of the rightmost particle of the system at time $n$. For almost all environment $\omega$, there exists a real number $S_{\omega}>0$ such that

$$
P_{\omega}\left(\liminf _{n \rightarrow \infty} m_{n}^{*} / n \geq S_{\omega}\right)>0 .
$$

Proof: We have, for $n \in \mathbb{N}, A \in \mathbb{N}$, and any even integer $N$, since $k_{\omega}$ is also even,

$$
\begin{aligned}
P_{\omega}\left\{\lambda\left([A,+\infty), n k_{\omega}+N\right)=0 \mid \mathcal{F}_{\omega}\left(n k_{\omega}\right)\right\} & =\prod_{x \in \mathbb{Z}} \prod_{\ell=1}^{\lambda\left(2 x, n k_{\omega}\right)} P_{\omega}^{2 x}\{\lambda([A,+\infty), N)=0\} \\
& \leq \prod_{x \in \mathbb{N}}\left(P_{\omega}^{2 x}\{\lambda([A,+\infty), N)=0\}\right)^{\lambda\left(2 x, n k_{\omega}\right)}
\end{aligned}
$$

By coupling, we have for $x \geq 0$,

$$
P_{\omega}^{2 x}\{\lambda([A,+\infty), N)=0\} \leq P_{\omega}^{0}\{\lambda([A,+\infty), N)=0\} .
$$


Thus,

$$
(9) \leq \prod_{x \in \mathbb{N}}\left(P_{\omega}^{0}\{\lambda([A,+\infty), N)=0\}\right)^{\lambda\left(2 x, n k_{\omega}\right)} \leq\left(P_{\omega}^{0}\{\lambda([A,+\infty), N)=0\}\right)^{Y_{n}} .
$$

Let $a \in(0,1)$ and $\varepsilon^{\prime}>0$. According to (5), there exists $M_{\omega} \in \mathbb{N}$ such that

$$
\forall N \geq M_{\omega}, \quad p_{\omega}(0 \rightsquigarrow[a N,+\infty), N) \geq \exp \left[-\left(I_{\eta}^{q}(a)+\varepsilon^{\prime}\right) N\right] \quad \mathbb{P} \text {-a.s. }
$$

If $q_{N}$ denotes the probability that the Galton-Watson tree $\Gamma$ extincts before time $N$, we notice that for $N \geq M_{\omega}$,

$$
\begin{aligned}
P_{\omega}^{0}(\lambda([a N,+\infty), N)=0) & \leq q_{N}+\left(1-q_{N}\right) p_{\omega}(0 \rightsquigarrow(-\infty, a N), N) \\
& \leq q_{N}+\left(1-q_{N}\right)\left\{1-\exp \left[-\left(I_{\eta}^{q}(a)+\varepsilon^{\prime}\right) N\right]\right\} \\
& =1-\left(1-q_{N}\right) \exp \left[-\left(I_{\eta}^{q}(a)+\varepsilon^{\prime}\right) N\right] .
\end{aligned}
$$

Let $E_{1}(\omega, n):=\left\{Y_{n} \geq 2^{n}\right\}$, and notice that $q_{N} \leq q_{\infty} \in[0,1)$. As a consequence, on $E_{1}(\omega, n)$, using (10) and (11), we obtain for $N \geq M_{\omega}$,

$$
\begin{aligned}
\log P_{\omega}\left\{\lambda\left([a N,+\infty), n k_{\omega}+N\right)=0 \mid F_{\omega}\left(n k_{\omega}\right)\right\} & \leq 2^{n} \log \left\{1-\left(1-q_{N}\right) \exp \left[-\left(I_{\eta}^{q}(a)+\varepsilon^{\prime}\right) N\right]\right\} \\
& \leq-2^{n}\left(1-q_{N}\right) \exp \left[-\left(I_{\eta}^{q}(a)+\varepsilon^{\prime}\right) N\right] \\
& \leq-\left(1-q_{\infty}\right) \exp \left[n \log 2-\left(I_{\eta}^{q}(a)+\varepsilon^{\prime}\right) N\right] .
\end{aligned}
$$

Let $N_{n}=2\left\lfloor\frac{n \log 2}{4\left(I_{\eta}^{q}(a)+\varepsilon^{\prime}\right)}\right\rfloor$. For all large $n$, we obtain on $E_{1}(\omega, n)$,

$$
P_{\omega}\left\{\lambda\left(\left[a N_{n},+\infty\right), n k_{\omega}+N_{n}\right)=0 \mid F_{\omega}\left(n k_{\omega}\right)\right\} \leq \exp \left\{-\left(1-q_{\infty}\right) C \exp [n(\log 2) / 2]\right\},
$$

where $C>0$ is a constant. Hence,

$$
P_{\omega}\left(\left\{\lambda\left(\left[a N_{n},+\infty\right), n k_{\omega}+N_{n}\right)=0\right\} \cap E_{1}(\omega, n)\right) \leq \exp \left\{-\left(1-q_{\infty}\right) C \exp [n(\log 2) / 2]\right\} .
$$

Consequently,

$$
\sum_{n \in \mathbb{N}} P_{\omega}\left(\left\{\lambda\left(\left[a N_{n},+\infty\right), n k_{\omega}+N_{n}\right)=0\right\} \cap E_{1}(\omega, n)\right)<+\infty .
$$

By Lemma 4.1, for almost all environment $\omega$ there exists an integer $n_{\omega}$ such that $P_{\omega}\left(E_{2}\left(\omega, n_{\omega}\right)\right)>0$, where $E_{2}(\omega, n):=\cap_{\ell \geq n} E_{1}(\omega, \ell)$. By the Borel-Cantelli lemma, we obtain $P_{\omega}-$ a.s. on $E_{2}\left(\omega, n_{\omega}\right)$, for $n$ large enough,

$$
\lambda\left(\left[a N_{n},+\infty\right), N_{n}+n k_{\omega}\right) \geq 1 .
$$

Then $P_{\omega}$-a.s. on $E_{2}\left(\omega, n_{\omega}\right)$, for all large $n$, there exists a particle $p_{n}$ in $\left[a N_{n},+\infty\right)$ at time $K_{n}:=N_{n}+n k_{\omega}$. At any time $\ell \in\left(K_{n-1}, K_{n}\right] \cap \mathbb{Z}$, the ancestor of the particle $p_{n}$ is located in $\left[a N_{n}-\left(K_{n}-K_{n-1}\right),+\infty\right)$, which is contained in $\left[S_{\omega} \ell,+\infty\right)$ for some constant $S_{\omega}>0$ (noticing that $K_{n}-K_{n-1}$ is bounded). Thus, for all large $\ell, \lambda\left(\left[S_{\omega} \ell,+\infty\right), \ell\right) \geq 1$. This means that $P_{\omega}-$ a.s. on $E_{2}\left(\omega, n_{\omega}\right)$ there are at any large time some particles with average speed greater than $S_{\omega}$. Since $P_{\omega}\left\{E_{2}\left(\omega, n_{\omega}\right)\right\}>0$, this completes the proof of the lemma.

\subsection{End of the proof}

For any $S>0$, we define the event $A(S):=\left\{\liminf _{n \rightarrow \infty} \frac{m_{n}^{*}}{n} \geq S\right\}$, where $m_{n}^{*}=-\infty$ if there is no particles left at time $n$. Let $\theta$ denote the shift operator, given by $(\theta \omega)_{i}=\omega_{i+1}$. The sequence $\left\{P_{\theta^{2 i} \omega}(A(S))\right\}_{i \in \mathbb{Z}}$ is a 
stationary sequence. Moreover, by a simple coupling argument, it is also a nondecreasing sequence. Thus it is constant, i.e. $\mathbb{P}$-a.s.,

$$
\forall i \in \mathbb{Z}, \quad P_{\theta^{2 i} \omega}(A(S))=P_{\omega}(A(S)) .
$$

By Lemma 4.2, for almost all environment $\omega$, there exists $S_{\omega}>0$ such that $P_{\omega}\left(A\left(S_{\omega}\right)\right)>0$. We now fix such an $\omega$ and set $S:=S_{\omega}$.

Let $1<r<m$. Let $B(N)$ denote the total number of particles at time $N$. Define $E_{3}(N):=\left\{B(N) \geq r^{N}\right\}$ for $N \in \mathbb{N}$. Notice that $\{\Gamma$ survives $\}=\liminf _{N \rightarrow \infty} E_{3}(N)$, since $\Gamma$ is a Galton-Watson tree such that $1<m<\infty$ and $\sigma^{2}<\infty$. On $E_{3}(2 N)$, with the convention $\sup \emptyset=-\infty$,

$$
P_{\omega}\left(\liminf _{n \rightarrow \infty} m_{n}^{*} / n<S \mid \mathcal{F}_{\omega}(2 N)\right) \leq \prod_{x \in \mathbb{Z}}\left\{1-P_{\theta^{2 x} \omega}[A(S)]\right\}^{\lambda(2 x, 2 N)} \leq\left\{1-P_{\omega}[A(S)]\right\}^{r^{2 N}}
$$

Consequently, since $P_{\omega}(A(S))>0$

$$
\lim _{N \rightarrow \infty} P_{\omega}\left(\left\{\liminf _{n \rightarrow \infty} m_{n}^{*} / n<S_{\omega}\right\} \cap E_{3}(2 N)\right)=0 .
$$

Since $\{\Gamma$ survives $\}=\liminf _{N \rightarrow \infty} E_{3}(N)$, this yields Part (ii) of Theorem 1.1.

\section{Proof of Proposition 1.2}

In the critical case $m=m_{c}>1$, we have $I_{\eta}^{q}(0)>0, v<0, I_{\eta}^{q}(v)=0$, and $I_{\eta}^{q}$ is strictly increasing on $[v, 1]$. Let $\alpha>0$. There exists $\varepsilon>0$ such that $\log m=I_{\eta}^{q}(0)<I_{\eta}^{q}(\alpha)-\varepsilon$. Thus as in $(6)$,

$$
\sum_{n \geq 0} P_{\omega}(\lambda((\alpha n,+\infty), n) \geq 1)<\infty
$$

for every $\alpha>0$ and almost every environment. As in Section 3, this gives (3).

Now, let $k>0$ be an even integer, and $A_{k}:=\left\{\omega, E_{\omega}(\lambda(\mathbb{N}, k))>1\right\}$. Suppose that $\mathbb{P}\left(A_{k}\right)>0$. For $\omega \in A_{k}$, we can build the tree $T$ and $Y_{n}$ as in Subsection 4.1, with $k$ instead of $k_{\omega}$. This is a supercritical Galton-Watson tree, and $E_{\omega}\left(Y_{n}\right)=a_{\omega}^{n}$ for $n \in \mathbb{N}$, where $a_{\omega}:=E_{\omega}(\lambda(\mathbb{N}, k))>1$. Let $\varepsilon>0$. For almost every $\omega \in A_{k}$, for $\varepsilon^{\prime}>0, N$ even and large enough, we have by coupling, with the same notation as in Subsection 4.1,

$$
\begin{aligned}
E_{\omega}(\lambda([\varepsilon N,+\infty[, N+n k)) & \geq E_{\omega}\left(Y_{n}\right) E_{\omega}(\lambda([\varepsilon N,+\infty[, N)) \\
& \geq a_{\omega}^{n}\left(1-q_{N}\right) P_{\omega}\left(X_{N} \geq \varepsilon N\right) \\
& \geq\left(1-q_{\infty}\right) \exp \left(\left(\log a_{\omega}\right) n-\left(I_{\eta}^{q}(\varepsilon)+\varepsilon^{\prime}\right) N\right) .
\end{aligned}
$$

If $\varepsilon^{\prime}$ is small enough, for $n=\left\lfloor\frac{2\left(I_{\eta}^{q}(e)+\varepsilon^{\prime}\right)}{\log \alpha_{\omega}} N\right\rfloor$, we have

$$
E_{\omega}(\lambda([\varepsilon N,+\infty[, N+n k)) \longrightarrow N \rightarrow+\infty+\infty .
$$

But for $N$ large enough, $n=\left\lfloor\frac{2\left(I_{\eta}^{q}(e)+\varepsilon^{\prime}\right)}{\log \alpha_{\omega}} N\right\rfloor, \varepsilon_{2}>0$ small enough and $\beta=\frac{2\left(I_{\eta}^{q}(\varepsilon)+\varepsilon^{\prime}\right)}{\log a_{\omega}} k+1$,

$$
\begin{aligned}
E_{\omega}(\lambda([\varepsilon N,+\infty[, N+n k)) & \leq m^{N+n k} P_{\omega}\left[X_{N+n k} \geq \varepsilon(N+n k) /(2 \beta)\right] \\
& \leq \exp \left[\left(I_{\eta}^{q}(0)-I_{\eta}^{q}(\varepsilon /(2 \beta))+\varepsilon_{2}\right)(N+n k)\right] \longrightarrow_{N \rightarrow \infty} 0 .
\end{aligned}
$$

This contradicts $(12)$. Hence, $E_{\omega}(\lambda(\mathbb{N}, 2 k)) \leq 1$ for every $k \in \mathbb{N}, \mathbb{P}$-a.s. 


\section{Comments}

6.A Another point of view for the model studied in this paper is to view the system of particles as a tree indexed process $\left(Y_{v}\right)_{v \in \Gamma}$. We set $Y_{\mathbf{o}}=0$, where $\mathbf{o}$ is the root of $\Gamma$. Moreover, for all $v \in \Gamma$, if the children of $v$ are denoted by $v_{1}, v_{2}, \ldots v_{n}$, we set for all $x \in \mathbb{Z}$,

$$
\begin{aligned}
& P_{\omega}\left(Y_{v_{1}}=Y_{v_{2}}=\cdots=Y_{v_{n}}=x+1 \mid \Gamma, Y_{v}=x\right)=\omega_{x}, \\
& P_{\omega}\left(Y_{v_{1}}=Y_{v_{2}}=\cdots=Y_{v_{n}}=x-1 \mid \Gamma, Y_{v}=x\right)=1-\omega_{x} .
\end{aligned}
$$

Furthermore, the locations of the offspring of two different vertices $u$ and $v$ of the same generation of $\Gamma$ are independent under $P_{\omega}$ conditionally on $\Gamma, Y_{u}$ and $Y_{v}$. One can formulate the proof of Theorem 1.1 in terms of $\left(Y_{v}\right)_{v \in \Gamma}$.

6.B Theorem 1.1 holds also for the following model: at time $t=0$ there is only one particle, located at 0 . At time $n$, each particle reproduces independently with the same law: it gives birth to $k$ offspring with probability $p_{k}$ and dies. Then each particle moves to a new location according to the transition probabilities $\left(\omega_{i}\right)_{i \in \mathbb{Z}}$. See for example Biggins (1977) in the case of usual random walks.

I am grateful to Zhan Shi for several helpful discussions, and to an anonymous referee for valuable comments. I also wish to thank Nina Gantert for sending me a version of Gantert et al. (1997).

\section{References}

Athreya, K.B. and Ney, P.E. (1972), Branching Processes. Springer, New York.

Benjamini, I. and Peres, Y. (1994), Tree-indexed random walks on groups and first passage percolation. Probab. Theory Related Fields 98, 91-112.

Biggins, J.D. (1977), Chernoff's theorem in the branching random walk. J. Appl. Probab. 14, 630-636.

Comets, F., Gantert, N. and Zeitouni, O. (2000), Quenched, annealed and functional large deviations for one-dimensional random walk in random environment. Probab. Theory Related Fields 118, 65-114.

Comets, F., Menshikov, M. V. and Popov, S.Y. (1998), One-dimensional branching random walk in a random environment: a classification. Markov Process. Related Fields, 4, 465-477.

Comets, F. and Popov, S.Y. (2005), On multimensional branching random walks in random environment. Preprint arXiv, math.PR/0507126.

Gantert, N. and Müller S. (2006), The critical Branching Markov Chain is transient. Markov Process. Related Fields, 12, 805-492.

Greven, A. and den Hollander, F. (1992), Branching random walk in random environment: phase transitions for local and global growth rates. Probab. Theory Related Fields 91, 195-249.

Greven, A. and den Hollander, F. (1994), Large deviations for a random walk in random environment. Ann. Probab 22, 1381-1428.

Hammersley, J.M. (1974), Postulates for subadditive processes. Ann. Probab. 2, 652-680.

Kingman, J.F.C. (1975), The first birth problem for an age-dependent branching process. Ann. Probab. 3, $790-801$.

Lyons, R. and Pemantle, R. (1992), Random walk in a random environment and first-passage percolation on trees. Ann. Probab. 20, 125-136.

Peres, Y. (1999), Probability on trees: an introductory climb. In: Lectures on Probability Theory and Statistics (St-Flour 1997), Lecture Notes in Math. 1717, pp. 193-280. Springer, Berlin.

Révész, P. (1994), Random Walks of Infinitely Many Particles. World Scientific, Singapore.

Solomon, F. (1975), Random walks in a random environment. Ann. Probab. 3, 1-31.

Zeitouni, O. (2004), Lecture notes on random walks in random environment. École d'été de probabilités de Saint-Flour 2001. Lecture Notes in Math. 1837, pp. 189-312. Springer, Berlin. 